Stefania Proietti

Thierry De Baere

Bertrand Bessoud

Francesco Doenz

Salah Dine Qanadli

Pierre Schnyder

Alban Denys

\title{
Interventional management of gastroduodenal lesions complicating intra-arterial hepatic chemotherapy
}

Received: 5 June 2006

Revised: 24 September 2006

Accepted: 28 November 2006

Published online: 12 January 2007

(C) Springer-Verlag 2007

S. Proietti $(\bowtie) \cdot$ F. Doenz

S. D. Qanadli · P. Schnyder · A. Denys

Department of Radiology,

Centre Hospitalier,

Universitaire Vaudois,

46 rue du Bugnon,

1011 Lausanne, Switzerland

e-mail: stefania.proietti@chuv.ch

Tel.: +41-21-3144586

Fax: +41-21-3144443

T. De Baere

Department of Interventional

Radiology, Institut Gustave-Roussy,

Villejuif, France

\section{B. Bessoud}

Department of Radiology,

Hôpital Bicêtre,

Le Kremlin-Bicêtre, France

\begin{abstract}
Herein we report the efficacy of embolization of small patent gastric or duodenal vessels for treating gastroduodenal complications after hepatic arterial infusion therapy (HAIC). Catheter ports were implanted percutaneously from a femoral approach in three cases or surgically in the gastroduodenal artery in two cases. Acute abdominal pain developed on average after four HAIC courses of 5FU-oxaliplatin, mytomycin, oxaliplatin or fotemustine. Esophagogastroduodenoscopy showed gastroduodenal lesions (gastroduodenitis with or without ulcerations) in all cases. Despite the interruption of the HAIC, symptoms persisted and led to selective hepatic arteriography showing a patent right gastric artery $(n=4)$ or a recanalized gastroduodenal artery $(n=1)$ responsible for gastroduodenal misper-
\end{abstract}

fusion. Successful embolizations of the arteries responsible for gastroduodenal misperfusion (right gastric artery in four cases and gastroduodenal artery in one case) using 0.018 platinium coils relieved the patients' symptoms and allowed the HAIC to continue. In gastroduodenal complications of HAIC, a selective hepatic arteriography should be performed to search any artery responsible for the misperfusion of the toxic agent in the gastroduodenal area. Embolization of these arteries allowed the HAIC to be restored.

\section{Keywords Liver metastasis .} Hepatic intra-arterial infusions . Chemotherapeutic infusions · Gastroduodenal lesions ·

Celiac arteriogram .

Angiographic embolization

\section{Introduction}

As compared to intravenous systemic chemotherapy, intraarterial hepatic chemotherapy has several theoretical advantages [1-3]. Because liver metastases receive most of their blood supply from the hepatic artery, they will be treated with higher drug concentrations with hepatic arterial infusion therapy (HAIC) than with systemic chemotherapy [1-3]. As compared to intravenous chemotherapy, concentrations of drugs in the tumor have been determined to be 5-20 times greater depending on the drug used [1]. Chemotherapeutic agents administered arterially will be extracted mainly by the liver, thus reducing systemic toxicity $[2,3]$.
Intra-arterial hepatic catheters can be placed surgically or percutaneously [4]. The most common site of surgically implanted catheter systems is the gastroduodenal artery (GDA) [5], whereas in the percutaneous approach a catheter is placed in the most suitable hepatic artery and may be associated with the embolization of the GDA and other aberrant hepatic arteries [4].

Gastroduodenal arterial blood supply not only arises from the celiac trunk and the splenic artery through the left gastric artery and the left gastro-epiploic artery, but also from small branches arising from the hepatic artery through the right gastric artery and duodenal arteries (Fig. 1). When these small branches are not ligated or embolized when the catheter is implanted, they may lead to inadvertent chemo- 


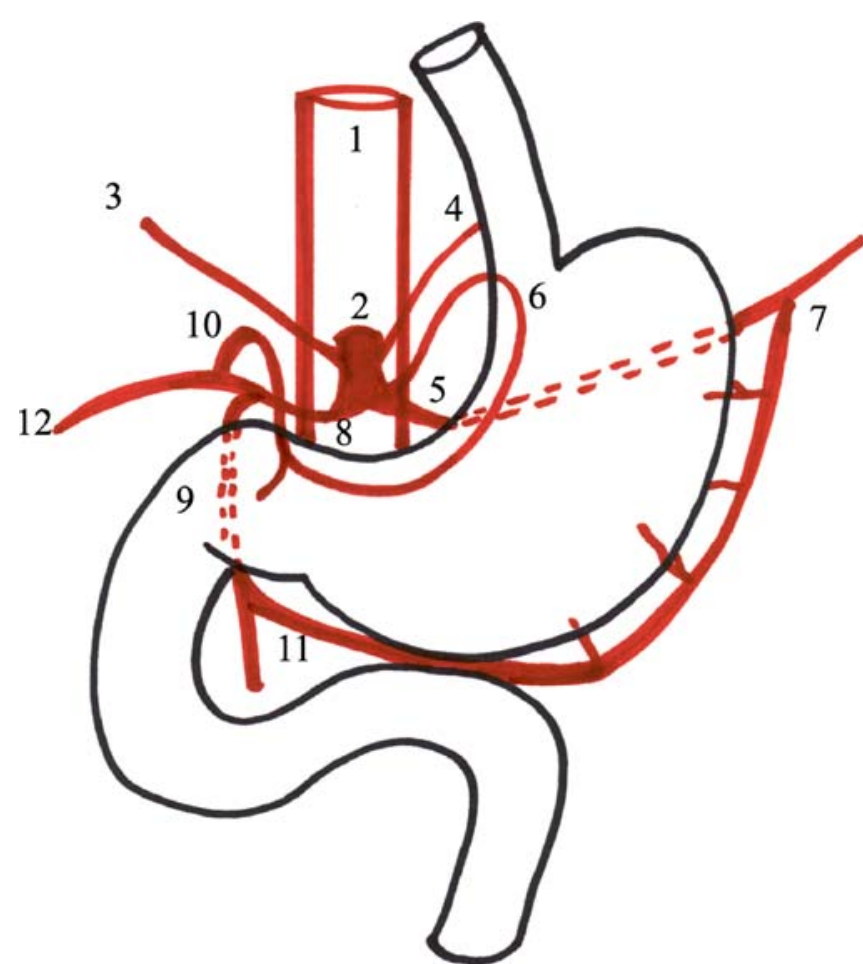

Fig. 1 Gastric arteries. 1 Aorta, 2 celiac trunk, 3 right inferior phrenic artery, 4 left inferior phrenic artery, 5 splenic artery, 6 left gastric artery, 7 left gastroepiploic artery, 8 trunk of the hepatic artery, 9 gastroduodenal artery, 10 right gastric artery, 11 right gastroepiploic artery, 12 hepatic artery

therapy perfusion of the gastroduodenal wall $[6,7]$. Gastroduodenal complications are refractory to medical treatments including antiacids and usually require interruption of HAIC [8].

We report five cases of documented gastroduodenal HAIC complications successfully treated by embolization of the arterial vessels responsible of gastroduodenal misperfusion. We describe the outcome of these patients and the possibility of continuing HAIC after embolization.

\section{Materials and methods}

Patients Out of 35 patients treated with HAIC for unresectable metastatic hepatic lesions in two institutions over a 15-month period, 5 patients (4 women and 1 man), aged 32-69 years (mean age of 56 years), developed gastroduodenal symptoms. All patients had biopsy-proven metastases originating from colorectal cancer $(n=3)$, epidermoid carcinoma of the anal canal $(n=1)$ and choroid melanoma $(n=1)$. None of our patients had previous history of gastroduodenal ulcers.

In three patients, the indwelling catheter-port system was implanted percutaneously via the femoral artery in the proper hepatic artery (B: Braun ST 305C, Emmen- brücke, Switzerland). We used the technique described by Herrmann et al. [4]. The common femoral artery is punctured using the Seldinger technique and the catheter advanced to the celiac trunk to assess the anatomy and possible variant hepatic arterial supply. No anatomical variation was encountered in our three patients. The catheter tip was placed into the GDA with the distal hole placed at the origin of the hepatic artery to allow for HAIC. The GDA was subsequently embolized by several coils. No complications occurred during the procedures.

The two other patients underwent open surgical implantation of the catheter [5]. Preoperative percutaneous hepatic arteriography allowed us to exclude any hepatic anatomical variation. The GDA was dissected and ligated $2-3 \mathrm{~cm}$ distally from its origin. In the proximal portion of the GDA, the catheter was fixed in order to have its extremity in the hepatic artery. The surgeons ligated the gastric and duodenal branches originating from the hepatic artery.

Both groups of patients underwent a nuclear-medicine liver perfusion scan before the infusion of chemotherapeutic agent in order to exclude any extrahepatic misperfusion.

HAIC courses consisted of 5FU-oxaliplatin (5FU: $1,000 \mathrm{mg} / \mathrm{m}^{2}$ and oxaliplatin: $85 \mathrm{mg} / \mathrm{m}^{2}$ once every 3 weeks), mytomycin ( $8 \mathrm{~g} / \mathrm{m}^{2}$ every 4.5 weeks), oxaliplatin $\left(100 \mathrm{mg} / \mathrm{m}^{2}\right.$ every 3 weeks) (for two patients) or fotemustine $\left(200 \mathrm{mg} / \mathrm{m}^{2}\right.$ every week for 4 weeks followed by $200 \mathrm{mg} / \mathrm{m}^{2}$ every 3 weeks).

Upper gastrointestinal symptoms consisted of persistent severe abdominal pain located in the right upper quadrant. Nausea or hematemesis was not encountered. Pain developed on average after four HAIC courses (range: two to six courses) and necessitated the interruption of the HAIC.

All patients underwent esophagogastroduodenoscopy showing gastroduodenal lesions. The lesions consisted of nonspecific inflammatory changes associated with $(n=4)$ or without $(n=1)$ ulcerative lesions. Bleeding was noted oozing from several points of the ulcer in one patient. The ulcerations were located in the gastric pyloric sphincter in four patients and also affected the duodenal bulb in one patient.

Upper abdominal pain was refractory to conservative treatment with omeprazole ( $20 \mathrm{mg}$ twice a day) for an average of 40 days. No antalgic treatment was reported.

An arteriography realized from the catheter-port system allowed the right position of the catheter to be checked in its original location and showed a patent RGA $(n=4)$ or a recanalized GDA $(n=1$, percutaneously implanted catheter associated with the gastroduodenal artery embolization) to be responsible for gastroduodenal misperfusion.

Procedures A 5-French catheter (Simmons 2 or Cobra 2, Glidecath, Terumo, Tokyo, Japan) was inserted from the right femoral artery in the celiac trunk to perform an angiography of hepatic arteries and branches. In four 

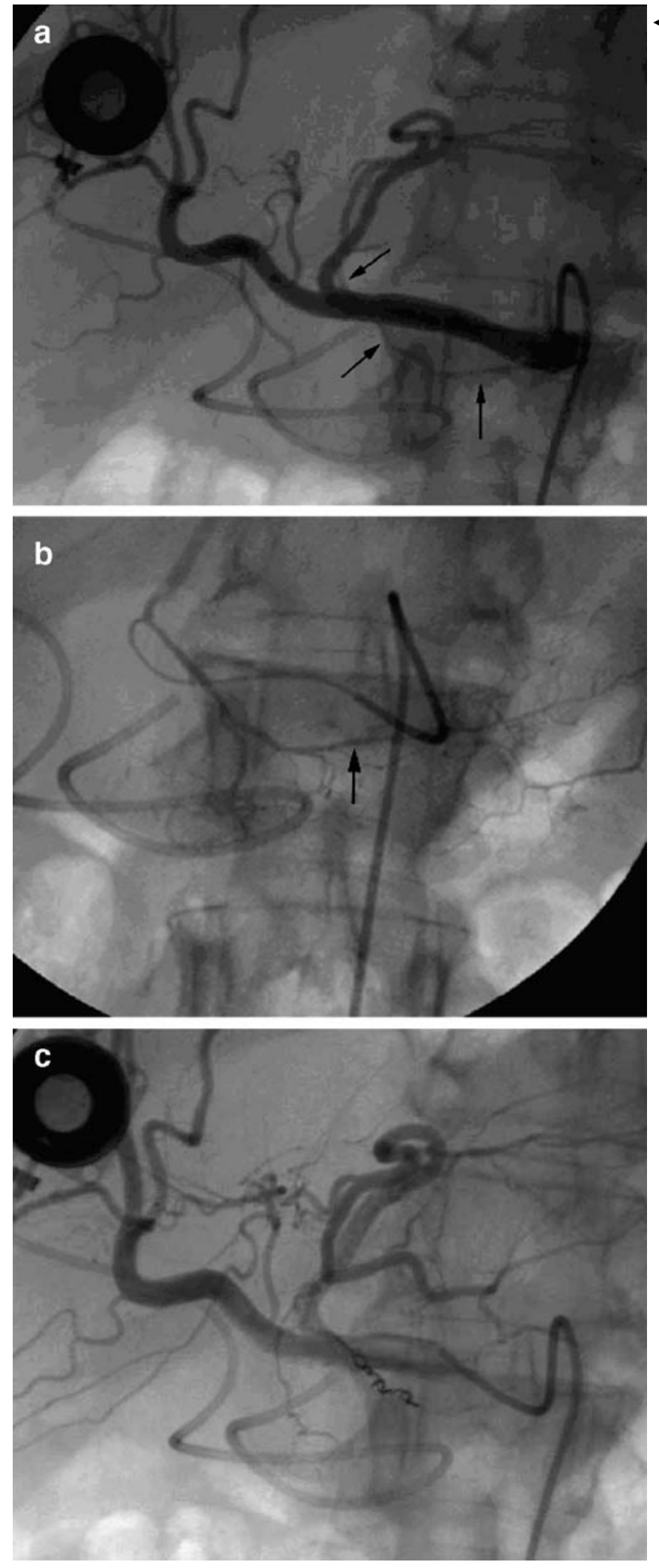

4Fig. 2a-c A 55-year-old man treated with HAIC for liver metastasis from colon cancer, presenting with epigastric pain. Intra-arterial port-catheter system was surgically implanted. a Celiac arteriogram with a Simmons 5-French catheter showing hepatic artery and its branches. Note that the right gastric artery (arrows) arises from the left hepatic branch. b Right gastric arteriogram (arrow) via microcatheter coaxially advanced from the 5-French catheter placed in the common hepatic artery. c Common hepatic arteriogram obtained after embolization of the right gastric artery by 3-2 mm Tornado soft platinum microcoils

patients a patent RGA originating from the hepatic artery and in one patient a recanalized GDA (percutaneously implanted catheter associated with the GDA embolization) were demonstrated.

A 2.7-French microcatheter (Progreat, Terumo, Europe N.V. Belgium) was inserted coaxially in the RGA $(n=4)$ or in the GDA $(n=1)$. Positioning in the RGA was performed anterogradely, via the left hepatic branch $(n=3)$ (Fig. 2) or retrogradely via the left gastric artery $(n=1)$ (Fig. 3). Embolization was done using 3-2 mm, 2-3 $\mathrm{mm}$, or 5-2 mm Tornado soft platinum microcoils (0.018 in) (Tornado, COOK Europe).

\section{Results}

Successful embolizations of the arteries responsible for gastroduodenal misperfusion (RGA in four cases and GDA in one case) relieved the patients and allowed the regional chemotherapy to be continued. Pain was relieved an average of 15 days (2-29 days) after embolization. The decision to resume HAIC was based on pain relief.

HAIC could be restarted for an average of 6.2 courses (2-10 courses).

None of the patients complained of further upper gastrointestinal symptoms.

Coil migration in the left hepatic artery occurred in one case, however the patient did not suffer left hepatic artery occlusion, and collaterals from the right hepatic artery were shown to have perfused the left lobe on the Technetium microspheres nuclear-medicine study.

One patient had to stop his intrahepatic chemotherapy because of a necrotizing cholangitis complicating HAIC after 12 courses, and the 4 other patients because of extrahepatic tumoral dissemination despite an hepatic tumoral lesion stabilisation.

\section{Discussion}

HAIC is a very efficient technique for the treatment of unresectable hepatic metastasis [1-3], but its use has been limited because of associated complications such as biliary 
Fig 3a-d A 32-year-old woman treated with HAIC for liver metastasis from choroid melanoma. Upper abdominal pain motivated an esophagogastroduodenoscopy that showed a $2.5-\mathrm{cm}$ gastric ulcer on the lesser curbure of the stomach. Intra-arterial portcatheter system was surgically implanted. a Celiac arteriogram with a Cobra 5-French catheter showing the hepatic artery and its branches. Note that the right gastric artery (arrow) can be seen. b Arteriogram obtained via 5 -French catheter placed in left gastric artery shows communication between left and right gastric arteries. c Microcatheter advanced into right gastric artery from left gastric artery. d Common hepatic arteriogram obtained after embolization of right gastric artery by 2-3 mm Tornado soft platinum microcoils (arrow)
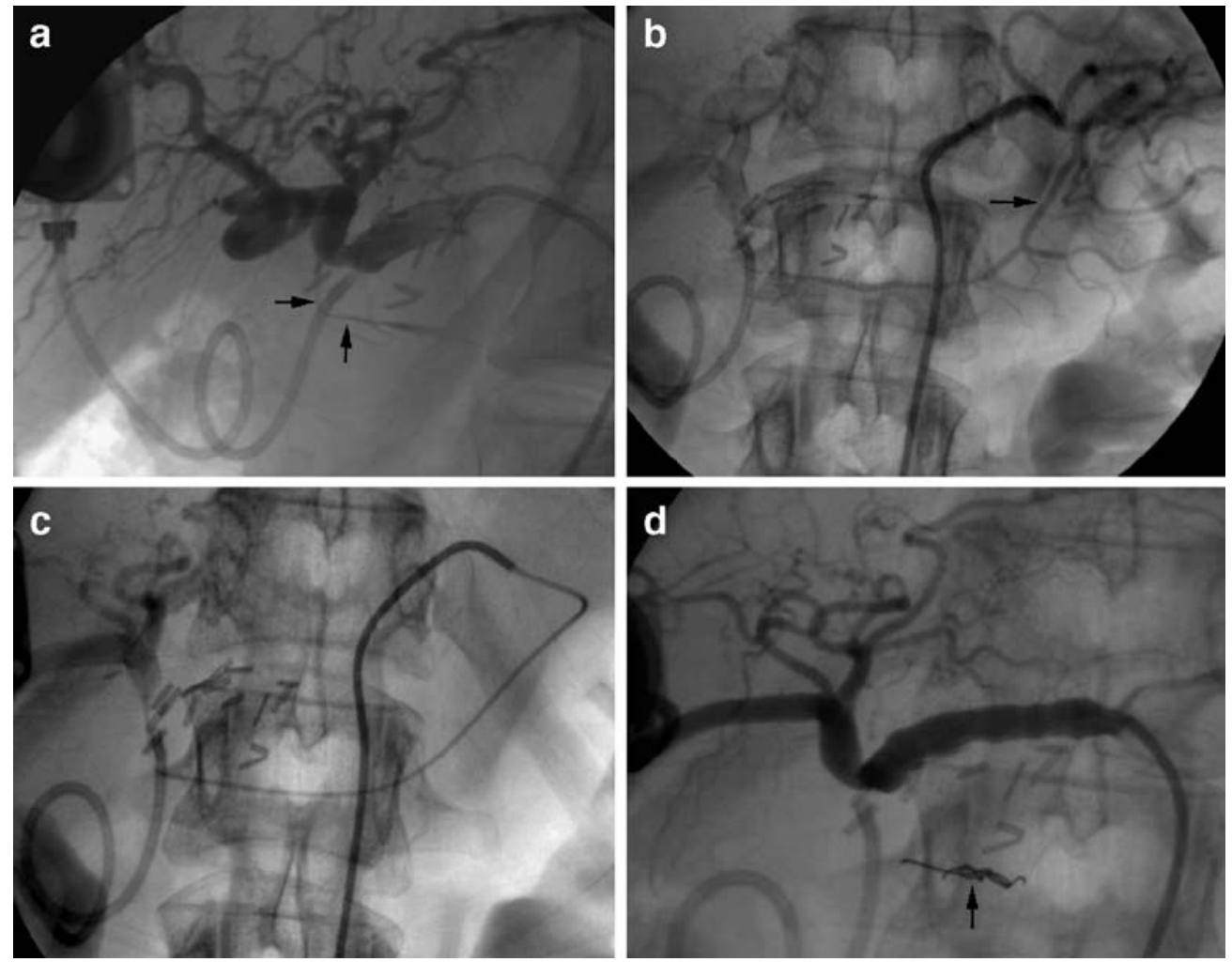

ischemic and/or toxic cholangiopathy, arterial occlusion or dissection, misperfusion of the agent and catheter dysfunction $[8,9]$. One of the most common complications, not encountered in patients treated with systemic chemotherapy [2, 3], is gastroduodenal ulcer, which occurs mainly after the first and second course of HAIC and which is considered to be a contraindication to continue [8]. Gastroduodenal complications were reported in $22 \%$ of patients in the series of Barnett et al. [9] reporting complications of HAIC in 4,580 patients.

GI lesions should be recognized as soon as possible because of the potentially fatal complications of perforation or upper gastrointestinal bleeding [10-12].

Esophagogastroduodenoscopy is strongly indicated to confirm the diagnosis in patients under HAIC who develop epigastric pain [12]. Gastroduodenal endoscopy allows the distribution of the ulcers in the territories of the misperfused arteries to be confirmed. The use of methylene blue administration via the arterial port during endoscopy has been used to establish gastroduodenal misperfusion from HAIC [13, 14]. Gastroduodenal abnormalities ranged from typical benign ulcers to a pattern of epithelial atypia and multiple ulcerations that may be falsely considered malignant [6]. Although the mucosal changes can be morphologically alarming, histological features indicative of HAIC-associated atypia have been established to differentiate it from carcinoma [7]. Chuang et al. [15] reported that 10 of the 18 patients (out of 174) presenting upper gastrointestinal pain had acute gastroduodenal lesions confirmed by endoscopy $(n=7)$ or upper gastroduodenal examination $(n=3)$ : 6 gastritis with gastric ulcers, 2 duodenal ulcers, 1 pyloroduodenitis and one pancreatitis. Endoscopically the iatrogenic ulceration and gastritis were located in the distribution of the infused arteries [15].

The pathogenesis of gastroduodenal complications after HAIC is probably multifactorial. The distribution of lesions follows vascular territories suggesting either an ischemic and/or a direct toxicity. These complications seem to be more frequent with 5-FU although they can be observed with other drugs [9] suggesting a predominantly toxic origin. Mucosal gastroduodenal ischemia has been related to the ligature or embolization of arterial supply and to the direct traumatic effect of the catheter and sutures in the intestinal wall $[10,16]$. Stress associated with the treatment may also play a role [10].

Treatment of these GI lesions is usually associated with antiulcer drugs as well as pain-control treatment, and HAIC is usually stopped because of the fear of recurrence $[8,10,12]$. In our series, embolization relieved pain and symptoms very quickly and allowed the HAIC treatment to be restored. This observation suggests that the toxic effect of drugs on the gastroduodenal wall is the most likely cause of these complications. Furthermore, embolization, which probably increases ischemia in this area, was surprisingly efficient for relieving symptoms. 
The surgical team of Hohn et al. [17] recommended ligature of all the small arteries originating from the hepatic artery and feeding the gastroduodenal region, GDA, RGA and also narrow arteries. None of their patients presented a gastrointestinal complication.

Song et al. [18] reported the importance of identification of nonhepatic arteries arising from the proper hepatic artery or from its distal branches before hepatic chemoembolization in order to adopt adequate preventive measures to avoid complications caused by nontarget perfusion. Embolization of the arteries responsible for misperfusion seems to be the most adequate approach. Although RGA embolization seems to present technical problems because of the RGA's relatively narrow lumen and its sharp branch $[15,19]$, Inaba et al. [19] described two techniques allowing successful embolization in 201 out of 217 patients. The embolization was performed either anterogradely via the hepatic artery (189 patients) or via the left gastric artery (12 patients).

Sofocleous et al. [20] reported 27 successful embolizations in 24 patients showing misperfusion on nuclearmedicine liver perfusion scan done within 2 weeks of surgical placement of the catheter tip in order to prevent any complication. We certainly agree with this approach. In our cases, when the catheter was placed, arteries allowing misperfusion were not recognized probably because of their small diameter. They may have increased in size afterwards because of the occlusion of the gastroduodenal artery related to catheter fixation.

Bloom et al. [14] reported 16 patients with liver metastasis from colorectal carcinoma and HAIC misperfusion. They separated the patients in two groups, extrahepatic $(n=8)$ and intrahepatic $(n=8)$ misperfusion. Five patients out of the extrahepatic group presented gastrointestinal symptoms associated with gastroduodenal misperfusion. This was confirmed by an endoscopy, performed simultaneously with methylene blue injection in the arterial port, whereupon the mucosa was observed to be stained blue. However no mucosal lesions were described. Three of these patients were sucessfully treated by percutaneous embolization of the vessels responsible for gastroduodenal misperfusion. The authors suggested that intrahepatic and extrahepatic misperfusion were caused mostly by inadequate angiographic evaluation of anatomical variants, failure to ligate vessels and to redistribute extrahepatic blood flow to collateral channels after catheter tip placement.

We must keep in mind that even with the pretreatment occlusion of the aberrant hepatic arteries, patients with hepatic tumors adjacent to the hepatic surface have the potential to develop collaterals to the liver during HAIC [21]. In the same way, newly developed abnormal branches may be responsible for gastroduodenal misperfusion, though gastroduodenal misperfusion may result from repermealisation of GDA and/or RGA or from newly developed collaterals to the stomach or the duodenum. Seki et al. [22] reported two cases of gastric toxicity related to HAIC, caused by the inflow of infused drugs via the left inferior phrenic artery arising from the celiac trunk, which was identified by digital subtraction angiography using the indwelling catheter. It is important to determine where the gastroduodenal misperfusion arises.

Gastroduodenal complications following HAIC should prompt arteriography of the hepatic arterial circulation. An evaluation to determine the presence of arteries that supply the gastroduodenal region that may be perfused during HAIC should be performed. If present, these vessels should be embolized as this may result in resolution of symptoms and continuation of HAIC.

To our knowledge this is the first clinical study describing the endovascular management of gastroduodenal documented lesions complicating HAIC, suggesting direct toxicity of the drug more than ischemic lesions. Furthermore in our study, this management allowed restoration of treatment increasing chances of survival for the patients.

\section{References}

1. Reed ML, Vaitkevicius VK, Al-Sarraf M et al (1981) The practicality of chronic hepatic artery infusion therapy of primary and metastatic hepatic malignancies: ten-year results of 124 patients in a prospective protocol. Cancer 47:402-409
2. Kemeny N, Daly J, Reichman B, Geller N, Botet J, Oderman P (1987) Intrahepatic or systemic infusion of fluorodeoxyuridine in patients with liver metastases from colorectal carcinoma. Ann Inter Med 107:459-465

3. Chang AE, Schneider PD, Sugarbaker PH (1987) A prospective randomized trial of regional versus systemic continuous 5-fluoroeoxyuridine chemotherapy in the treatment of colorectal liver metastases. Ann Surg 206:685691
4. Herrmann KA, Waggershauser T, Sittek H, Reiser MF (2000) Liver intraarterial chemotherapy: use of the femoral artery for percutaneous implantation of catheterport systems. Radiology 215(1):294-299 
5. Aldrighetti L, Arru M, Angeli E, Venturini M, Salvioni M, Ronzoni M, Caterini R, Ferla G (2002) Percutaneous vs. surgical placement of hepatic artery indwelling catheters for regional chemotherapy. Hepatogastroenterology 49(44):513-517

6. Doria MI Jr, Doria LK, Faintuch J, Levin B (1994) Gastric mucosal injury after hepatic arterial infusion chemotherapy with fluoxuridine. A clinical and pathologic study. Cancer 73 (8):2042-2047

7. Petras RE, Hart WR, Bukowski RM (1985) Gastric epithelial atypia associated with hepatic arterial infusion chemotherapy. Its distinction from early gastric carcinoma. Cancer 56(4):745750

8. Ravizza D, Fazio N, Crosta C, Fiori G, Orsi F, Grasso R, Medici M, Ferretti G, de Braud F (2003) Iatrogenic gastroduodenal ulcers during hepatic intra-arterial chemotherapy. Hepatogastroenterology 50:49-53

9. Barnett KT, Malafa MP (2001) Complications of hepatic artery infusion: a review of 4580 reported cases. Int $\mathrm{J}$ Gastrointest Cancer 30(3):147-160

10. Sundqvist K, Hafström Lo, Jönsson P-E, Lunderquist A, Nowak E (1983) Upper G-I bleeding following intraarterial chemotherapy infusion. Ann Chir Gynaecol 72:183-186
11. Narsete T, Ansfield F, Wirtanen G, Ramirez G, Wolberg W, Jarrett F (1977) Gastric ulceration in patients receiving intrahepatic infusion of 5-fluorouracil. Ann Surg 186(6): 734-736, Dec

12. Hinshaw KA, Bouwman DL, Weaver DW, Kinzie J (1986) Upper gastrointestinal endoscopy for diagnosis of complications of regional hepatic chemotherapy. Am Surgeon 52(7):351-353

13. Frye JW, Venook AP, Ostroff JW, Mulvihill SJ, Warren RS, Pellegrini CA, Ring EJ, Stagg RJ (1992) Hepatic intra-arterial methylene blue injection during endoscopy: a method of detecting gastroduodenal misperfusion in patients receiving hepatic intra-arterial chemotherapy via an implanted pump. Gastrointest Endosc 38(1):52-54

14. Bloom AI, Gordon RL, Ahl KH, Kerlan RK Jr, LaBerge JM, Wilson MW, Venook AP, Warren R (1999) Transcatheter embolization for the treatment of misperfusion after hepatic artery chemoinfusion pump implantation. Ann Surg Oncol 6(4):350-358, Jun

15. Chuang VP, Wallace S, Stroehlein J, Yap HY, Patt YZ (1981) Hepatic artery infusion chemotherapy: gastroduodenal complications. Am J Roentgenol 137 (2):347-350

16. Kernstine KH, Kryjeski SR, Hall LJ, Larson RE, Saylor HL 3rd, Fortuny IE (1990) Gastroduodenal artery-duodenal fistula: a complication of continuous floxurudine (FUDR) infusion into the gastroduodenal artery. J Surg Oncol 45 (1):59-62
17. Hohn DC, Stagg RJ, Price DC, Lewis BJ (1985) Avoidance of gastroduodenal toxicity in patients receiving hepatic arterial 5-fluoro-2'-deoxyuridine. J Clin Oncol 3(9): 1257-1260

18. Song SY, Chung JW, Lim HG, Park JH (2006) Nonhepatic arteries originating from the hepatic arteries: angiographic analysis in 250 patients. J Vasc Interv Radiol 17(3):461-469

19. Inaba Y, Arai Y, Matsueda K, Takeuchi Y, Aramaki T (2001) Right gastric artery embolization to prevent acute gastric mucosal lesions in patients undergoing repeat hepatic arterial infusion chemotherapy. Vasc Interv Radiol 12(8):957-963

20. Sofocleous CT, Schubert J, Kemeny N, Covey AM, Brody LA, Getrajdman GI, Thornton R, Winston C, Brown KT (2006) Arterial embolization for salvage of hepatic artery infusion pumps. J Vasc Interv Radiol 17(5):801-806

21. Seki H, Kimura M, Yoshimura N, Yamamoto S, Ozaki T, Sakai K (1998) Development of extrahepatic arterial blood supply to the liver during hepatic arterial infusion chemotherapy. Eur Radiol 8:1613-1618

22. Seki H, Kimura M, Yoshimura N, Yamamoto S, Ozaki T, Sakai K (1999) Gastric toxicity related to perfusion of the stomach via the left inferior phrenic artery during hepatic arterial infusion chemotherapy: report of two cases. Radiat Med 17(6):435-438 